\title{
Anti-VEGF drug interference with VEGF quantitation in the R\&D systems human quantikine VEGF ELISA kit
}

\author{
Giane Sumner ${ }^{1}$, Camille Georgaros ${ }^{1}$, Ashique Rafique ${ }^{1}$, Thomas DiCioccio ${ }^{1}$, Joel Martin ${ }^{1}$, \\ Nicholas Papadopoulos ${ }^{1}$, Thomas Daly ${ }^{1}$ \& Albert Torri*,1 \\ ${ }^{1}$ Regeneron Pharmaceuticals, Inc., Tarrytown, NY 10591, USA \\ *Author for correspondence: Tel.: +1 914847 7836; Albert.Torri@regeneron.com
}

\begin{abstract}
Aim: To evaluate the accuracy of the Quantikine Human VEGF Immunoassay (R\&D Systems) in the presence of VEGF inhibitors. Materials \& Methods: Quantikine VEGF ELISA (R\&D), anti-VEGF 165 mAb (R\&D), VEGF $_{165}$ and aflibercept (Regeneron), ranibizumab and bevacizumab (Genentech). Results: Binding affinity of anti-VEGF 165 mAb for VEGF was threefold weaker than aflibercept, but 33- and 40-fold stronger than ranibizumab or bevacizumab. Extended incubation of VEGF complexed with inhibitors led to VEGF dissociation from ranibizumab and bevacizumab, but not aflibercept, and subsequent binding by the immunoassay capture antibody. The immunoassay also detected VEGF:ranibizumab and VEGF:bevacizumab complexes but not VEGF:aflibercept complexes. Conclusion: The immunoassay cannot accurately quantitate VEGF in the presence of these VEGF inhibitors as they interfere with the capture and detection of free VEGF.
\end{abstract}

First draft submitted: 6 April 2018; Accepted for publication: 22 January 2019; Published online: 20 March 2019

Keywords: aflibercept $\bullet$ anti-VEGF drug $\bullet$ bevacizumab $\bullet$ ELISA $\bullet$ ranibizumab $\bullet$ VEGF $\bullet$ VEGF-A $\bullet$ VEGF inhibitor

VEGF is a signaling protein produced by cells, which stimulates angiogenesis, resulting in increased vascularization and vessel permeability. The physiological role of VEGF is to create new blood vessels. Unlike an endocrine hormone such as insulin, which is produced in one organ and disseminated broadly through the blood circulation in sufficient quantities to influence multiple other organs, VEGF is an autocoid, which is produced and acts locally in vascular tissues [1]. VEGF is activated locally in response to pathology, for example, to guide vessel regrowth at sites of injury or promote collateralization in ischemic tissue. Overexpression of VEGF in the eye can lead to neovascularization of the retina and the choroid and lead to various ocular diseases, such as neovascular age-related macular degeneration (AMD) [2-5].

Multiple VEGF inhibitors, including aflibercept, ranibizumab and bevacizumab, are being used as therapies to treat VEGF-related eye diseases (such as AMD, retinal vein occlusion and diabetic macular edema). Two of these anti-VEGF therapies, aflibercept and ranibizumab, were specifically developed to be administered intravitreally (IVT) and have been approved for these indications [5,6]. Bevacizumab is used off-label [7]. Aflibercept is a 115-kDa recombinant, dimeric glycoprotein. It is a soluble decoy receptor comprised of the fused extracellular domains 2 and 3 from VEGFR1 and VEGFR2, respectively, linked to the Fc portion of human IgG1 [8]. Ranibizumab is a 48-kDa monovalent monoclonal antibody $(\mathrm{mAb})$ antigen-binding $(\mathrm{Fab})$ fragment without the Fc domain. Bevacizumab is a $149-\mathrm{kDa}$ intact $\mathrm{mAb}$. Since each of these VEGF inhibitors is administered IVT into the eye, systemic exposure is minimal and is not expected to be clinically relevant.

VEGF is produced at a relatively high rate in the body $(25 \mathrm{ng} / \mathrm{g}$ of tissue/day) as required by the role it plays in the vasculature [9]. Since VEGF contains a heparin-binding domain, most of the VEGF produced by the body is bound to heparan sulfate on endothelial cell surfaces and not found in circulation. This explains the low levels of circulating VEGF in plasma, which ranges from undetectable in most individuals to low concentrations ranging from approximately 10 to $55 \mathrm{pg} / \mathrm{ml}[10,11]$. Furthermore, physiologic mechanisms restrict the activity of VEGF to its intended site of action, in other words, platelets sequester VEGF released into the systemic circulation [10]. 
The small amount of VEGF inhibitor that might be expected to be released into the systemic circulation following intravitreal injection would only bind to a small fraction of the VEGF produced daily by the body.

All three VEGF inhibitors bind tightly to VEGF. However, based on surface plasmon resonance (SPR) analysis, aflibercept has a higher binding affinity (binding constant $\left[K_{D}\right]$ ) for VEGF than the other two VEGF inhibitors (aflibercept $0.49 \mathrm{pM}$, ranibizumab $46 \mathrm{pM}$ and bevacizumab $58 \mathrm{pM}$ ) [12]. These binding affinities determined by SPR were supported by solution-based equilibrium-binding experiments using KinExA technology to avoid any potential binding artifacts, resulting from a surface-based approach and by experiments conducted in four different types of bioassay systems [12]. These VEGF inhibitors also differ with respect to their stoichiometry of VEGF binding [13]. Aflibercept can only bind to a single molecule of VEGF, whereas bevacizumab can bind to two molecules of VEGF. In addition, due to the dimeric nature of VEGF, two drug molecules can bind to one VEGF molecule in the case of bevacizumab or ranibizumab, leading to the formation of multimeric high-molecular-weight complexes with VEGF in both cases and the formation of extended chains (paper doll structures) in the case of bevacizumab [13]. In contrast, due to its unique structure and binding mechanism, only one VEGF molecule can bind to aflibercept, thus it only forms 1:1 molar complexes with VEGF [8].

Several recent studies that evaluated circulating VEGF levels following administration of VEGF inhibitors [14-17] suggested that IVT administration of aflibercept may decrease circulating VEGF concentrations to a greater extent than IVT administration of bevacizumab or ranibizumab. In one study, plasma concentrations of VEGF were measured in 56 patients with AMD following 3 monthly IVT administrations of aflibercept $(2 \mathrm{mg})$, ranibizumab $(0.5 \mathrm{mg})$ or bevacizumab $(1.25 \mathrm{mg})$ [15]. The authors reported a median $t_{\max }$ for total aflibercept, ranibizumab and bevacizumab of 1.1, 0.9 and 7.0 days, respectively, and reported a $\mathrm{C}_{\max }$ after the first dose of 51.8, 5.3 and $113.2 \mathrm{ng} / \mathrm{ml}$, respectively $(0.45,0.11$ and $0.76 \mathrm{nM}$, respectively). Mean baseline levels of free VEGF ranged from 17.0 to $22.5 \mathrm{pg} / \mathrm{ml}(0.87-1.15 \mathrm{pM})$. Therefore, all three VEGF inhibitors are in vast molar excess relative to circulating free VEGF levels. However, they reported that mean levels of free VEGF, after a single IVT administration of $2 \mathrm{mg}$ aflibercept, fell below the limit of detection $(10 \mathrm{pg} / \mathrm{ml})$, while plasma concentrations of free VEGF stayed at or above the limit of detection after a single IVT administration of ranibizumab $(14.4 \mathrm{pg} / \mathrm{ml})$ or bevacizumab $(10 \mathrm{pg} / \mathrm{ml})$. Similar results were reported in three other studies $[14,16,17]$. The authors of these papers have suggested that IVT administration of aflibercept may decrease circulating VEGF concentrations and that a reduction in circulating VEGF might have clinical consequences.

Each of these four studies measured circulating VEGF concentrations in patient samples using the commercially available R\&D Systems Quantikine Human VEGF ELISA kit (referred to as Quantikine VEGF ELISA hereafter), which has a lower limit of sensitivity of $9 \mathrm{pg} / \mathrm{ml}(0.23 \mathrm{pM})$ [11]. However, Takahashi et al. [18] reported that VEGF concentrations measured in a solution of known VEGF concentration $(1000 \mathrm{pg} / \mathrm{ml})$ were underestimated by the Quantikine VEGF ELISA in the presence of VEGF inhibitors, aflibercept, bevacizumab and ranibizumab, but not pegaptanib. In this paper, the authors cautioned that this assay does not accurately quantitate VEGF in the presence of these inhibitors and postulated that these VEGF inhibitors competed with the ELISA antibody to bind to VEGF. Therefore, understanding how aflibercept, bevacizumab and ranibizumab may interfere with the measurement of VEGF using the Quantikine VEGF ELISA is critical for the interpretation of data generated using this kit to measure VEGF levels in clinical samples containing these VEGF inhibitors.

In a separate study [19], using a different methodology to measure VEGF, only a modest and temporary decrease in circulating VEGF concentration was observed in 16 patients after IVT administration of $2 \mathrm{mg}$ aflibercept. In this study, the mean free VEGF concentration was $59.6 \mathrm{pg} / \mathrm{ml}$ at baseline and only decreased to $32.5 \mathrm{pg} / \mathrm{ml}$ after administration of aflibercept. Although no direct comparisons with other VEGF inhibitors were performed in this study, the results indicated that circulating VEGF remained at detectable levels after IVT administration of aflibercept and the concentrations reported were much higher than those reported in the other four studies previously described [14-17]. The principal difference between this study and the other studies was the use of a different commercially available assay (Bio-Rad Bio-Plex Cytokine VEGF-A Luminex assay) to measure circulating VEGF concentrations. The Luminex assay uses a capture antibody on soluble beads that increases the surface area for the assay. These results demonstrate that an alternative method can yield different results. The method used for quantifying VEGF should be examined thoroughly to ensure it functions appropriately under the conditions used (e.g., in the presence of VEGF inhibitors) and accurately generates reproducible and meaningful results.

Here, we examined whether the presence of the VEGF inhibitors, which are in approximately 100-fold molar excess of the circulating VEGF, affects the detection of VEGF in the Quantikine VEGF ELISA. The Quantikine VEGF ELISA utilizes a specific anti-VEGF $165 \mathrm{mAb}$ as the capture reagent. As such, the presence of VEGF-binding 
molecules (e.g., VEGF inhibitors) in a plasma sample could compete with the ELISA capture reagent for binding to free VEGF in the plasma sample. The degree of this type of assay interference would depend on the relative binding affinities between the VEGF inhibitors and the anti-VEGF ELISA capture reagent for VEGF. Therefore, the binding affinity of each of the VEGF inhibitors for VEGF is relevant for the interpretation of Quantikine VEGF ELISA results. Aflibercept was previously demonstrated to possess an approximately 100 -fold greater binding affinity $\left(\mathrm{K}_{\mathrm{D}} \sim 0.49 \mathrm{pM}\right)$ for VEGF- $\mathrm{A}_{165}$, relative to the binding affinities for either ranibizumab $\left(\mathrm{K}_{\mathrm{D}} \sim 46 \mathrm{pM}\right)$ or bevacizumab $\left(K_{\mathrm{D}} \sim 58 \mathrm{pM}\right)$ [12]. Therefore, aflibercept would be expected to compete more effectively with the anti-VEGF-A $\mathrm{A}_{165}$ capture antibody in the Quantikine VEGF ELISA for binding to VEGF in the patient plasma sample, compared with either ranibizumab or bevacizumab. As a result of these differential binding affinities for VEGF between the three VEGF inhibitors, the detection of free VEGF by the Quantikine VEGF ELISA is expected to vary depending on the specific VEGF inhibitor present in the sample.

To better understand the potential for interference in the Quantikine VEGF ELISA resulting from the presence of VEGF inhibitors in the patient plasma samples, a series of competitive binding experiments were conducted. First, the binding affinity of the anti-VEGF 165 mAb (MAB293 from R\&D Systems), the putative capture antibody used in the Quantikine VEGF ELISA, was determined. The relative binding affinities of each VEGF inhibitor for VEGF could then be compared with the binding affinity of the anti-VEGF $165 \mathrm{mAb}$. Second, using a known sample with a fixed concentration of VEGF, the percent analyte (VEGF) recovery (\%AR) of the Quantikine VEGF ELISA was determined in the presence of increasing concentrations of each inhibitor: aflibercept, ranibizumab and bevacizumab. Third, we examined whether the Quantikine VEGF ELISA measures only free VEGF or free VEGF and VEGF complexed with VEGF inhibitors. Samples with a fixed VEGF concentration in the presence of increasing concentrations of VEGF inhibitors were analyzed in the kit using specific anti-human IgG antibodies as the detection reagents (replacing the anti-VEGF polyclonal antibody supplied in the Quantikine VEGF ELISA).

The results demonstrate that the presence of VEGF inhibitors can interfere with the Quantikine VEGF ELISA and the level of this interference is directly related to the relative binding affinities of these inhibitors for VEGF. Furthermore, the results obtained with the anti-human IgG antibodies suggest that the Quantikine VEGF ELISA kit also detects VEGF complexed with either ranibizumab or bevacizumab, however, it does not detect VEGF complexed with aflibercept. Thus, 'free' VEGF measurements obtained in the presence of either ranibizumab or bevacizumab may be overestimated and include VEGF in complex with these inhibitors, further distorting the results obtained using the Quantikine VEGF ELISA.

\section{Materials \& methods VEGF reagents}

Human VEGF 165 (REGN110, Regeneron Pharmaceuticals, Inc., NY, USA).

\section{VEGF inhibitors}

Eylea (aflibercept, VEGF Trap, Regeneron Pharmaceuticals, Inc.), Lucentis (ranibizumab, Genentech, CA, USA), Avastin (bevacizumab, Genentech).

\section{Detection reagents}

Quantikine ELISA Human VEGF Immunoassay Kit (R\&D Systems, MN, USA). Peroxidase-conjugated AffiniPure goat antihuman IgG, Fc $\gamma$ fragment-specific (minimal cross-reaction to bovine, horse and mouse serum proteins; Jackson ImmunoResearch, PA, USA). Peroxidase-conjugated AffiniPure goat antihuman IgG, $\mathrm{F}(\mathrm{ab})_{2}$ fragmentspecific (minimal cross-reaction to bovine, horse and mouse serum proteins; Jackson ImmunoResearch).

\section{Wash \& buffers}

Wash solution concentration 20× (Kirkegaard and Perry Laboratories, MD, USA), 10\% bovine serum albumin, diluent/blocking solution (Kirkegaard and Perry Laboratories), Surfact-Amps 20 (ThermoFisher Scientific/Pierce, MA, USA), 10× Dulbecco's phosphate-buffered saline (without calcium chloride and magnesium chloride (ThermoFisher Scientific/Life Technologies, MA, USA).

Determination of kinetic-binding parameters for the anti-VEGF $165 \mathrm{mAb}$

SPR experiments were performed on a Biacore 3000 instrument using a dextran-coated (CM5) chip at $25^{\circ} \mathrm{C}$. The running buffer was filtered Hepes-buffered saline (10 mM Hepes, $150 \mathrm{mM} \mathrm{NaCl}, 3.4 \mathrm{mM}$ EDTA, 0.05\% 
polysorbate 20, $\mathrm{pH}$ 7.4). A capture sensor surface was prepared by covalently immobilizing either recombinant Protein A (Pierce, IL, USA), an anti-human Fab polyclonal antibody (human Fab capture kit, GE Healthcare, NJ, USA) or an anti-mouse Fc $\gamma$ (Jackson ImmunoResearch) to the chip surface through amine-coupling chemistry (1-ethyl-3-[3-dimethylaminopropyl] carbodiimide hydrochloride)/ $N$-hydroxysuccinimide [EDC/NHS]).

Aflibercept and bevacizumab were captured on the coupled Protein A chip surface, while ranibizumab was captured on the coupled anti-human Fab polyclonal antibody chip surface [12]. The anti-human/primate VEGF ${ }_{165}$ $\mathrm{mAb}$ (MAB293 [ $\operatorname{IgG}_{2 \mathrm{~B}}$ clone \#26503 for the immunogen S. frugiperda ovarian cell line 21 (Sf21) recombinant human $\left.V_{E G F}{ }_{165}\right]$ from R\&D Systems), believed to be the Quantikine ELISA capture reagent, was captured on the coupled anti-mouse Fc $\gamma$ chip surface. Following the capture step, varying concentrations of test ligand (20$0.062 \mathrm{nM}$ for VEGF- $\mathrm{A}_{165}$ ligand) were individually injected over the prepared anti-VEGF- $\mathrm{A}_{165}$ capture reagent surface or the surfaces prepared with the three VEGF inhibitor therapeutics. The association rate constant $\left(\mathrm{k}_{\mathrm{a}}\right)$ was determined from data obtained at multiple test ligand (VEGF-A $A_{165}$ ) concentrations. The dissociation rate constant $\left(k_{d}\right)$, which is independent of ligand concentration, was determined from the change in resonance units signal resulting from dissociation of VEGF- $\mathrm{A}_{165}$ from each bound VEGF inhibitor: VEGF- $\mathrm{A}_{165}$ ligand sample dissociation time was approximately $1-3 \mathrm{~h}$. Specific Biacore kinetic sensorgrams were obtained using a double referencing procedure. The data were then processed using Scrubber software (version 2.0, BioLogic Software, Seyssinet-Pariset, France) and kinetic analyses were performed using BiaEvaluation (version 4.1, Biacore, Uppsala, Sweden). The equilibrium dissociation constant $\left(\mathrm{K}_{\mathrm{d}}\right)$ was calculated from the ratio of the dissociation rate constant divided by the association (binding) rate constant $\left(K_{D}=k_{d} / k_{a}\right)$.

\section{Determination of anti-VEGF drug interference in the quantikine VEGF ELISA}

The Quantikine VEGF ELISA (R\&D Systems) was performed as per manufacturer's instructions [11], unless otherwise specified. The microplates provided with the kit were precoated with a mAb specific for Sf21-expressed recombinant human VEGF $_{165}$ (capture reagent) [11]. This recombinant human VEGF 165 was also provided in the kit as the positive control. The manufacturer claims that the recombinant and naturally occurring VEGF behave similarly in the assay. Briefly, the Assay Diluent (a buffered protein base) was added to the microplate (100 $\mu \mathrm{l} /$ well), followed by the addition of VEGF standards or samples $(100 \mu \mathrm{l} /$ well). The microplates were incubated for $2 \mathrm{~h}$ at room temperature and then washed three times with $400 \mu \mathrm{l}$ /well of wash buffer $(2 \mathrm{mM}$ imidazole, $160 \mathrm{mM} \mathrm{NaCl}$, $0.02 \%$ Tween 20, $0.5 \mathrm{mM}$ EDTA). The detection reagent, a peroxidase-conjugated anti-VEGF polyclonal antibody, was added to the microplates $(200 \mu \mathrm{l} /$ well). The microplates were incubated for $2 \mathrm{~h}$ at room temperature and then washed three times ( $400 \mu \mathrm{l} /$ well of wash buffer). The peroxidase-specific substrate was then added $(200 \mu \mathrm{l} /$ well). After approximately $20 \mathrm{~min}$, Stop solution $(2 \mathrm{~N}$ sulfuric acid) was added to the microplates $(50 \mu \mathrm{l} /$ well) to stop the peroxidase reaction and achieve a color intensity that was proportional to the concentration of VEGF.

The potential interference of three VEGF inhibitors (aflibercept, ranibizumab and bevacizumab) in the quantitation of VEGF was examined by preparing samples containing $1.3 \mathrm{pM}(50 \mathrm{pg} / \mathrm{ml})$ of VEGF with increasing concentrations of each inhibitor, ranging from 2000 to $0.005 \mathrm{pM}$ in buffer $(0.5 \%$ bovine serum albumin, $0.05 \%$ Tween 20, $1 \times$ Dulbecco's phosphate-buffered saline). The samples were incubated at room temperature for approximately 3 days (67-70 h) to allow VEGF:VEGF inhibitor complexes to form and samples to reach equilibrium prior to analysis, to mimic in vivo conditions where low concentrations of VEGF:VEGF inhibitor complexes are found in circulation after IVT administration. Based on the known $\mathrm{K}_{\mathrm{D}}$ 's for the VEGF:VEGF inhibitor complexes and the concentrations evaluated, a 3-day incubation was conducted to ensure complex formation with each VEGF inhibitor was complete [12]. Samples were then analyzed following either a 2-h sample incubation on the plate (normal kit conditions) or an extended overnight sample incubation $(\sim 20 \mathrm{~h})$ at room temperature. The concentration of VEGF chosen for the study $(1.3 \mathrm{pM}[50 \mathrm{pg} / \mathrm{ml}])$ was similar to reported concentrations in plasma $(40-60 \mathrm{pg} / \mathrm{ml}[9])$. However, these levels of VEGF are at the lower limit of the sensitivity of the R\&D ELISA using the manufacturers standard curve (lowest standard of $31.3 \mathrm{pg} / \mathrm{ml}[0.81 \mathrm{pM}]$ ). Therefore, additional experiments were also performed with samples containing $13 \mathrm{pM}(500 \mathrm{pg} / \mathrm{ml})$ of VEGF with increasing concentrations of each of the three VEGF inhibitors, ranging from 2000 to $0.005 \mathrm{pM}$, in order to generate data with greater signal-to-noise ratios. The mean optical density (450-570) readings for the VEGF standards were calibrated against their respective nominal concentrations using a 4-Parameter Logistic nonlinear regression fit. The resulting standard curve was used to compute the concentration of VEGF detected using the Quantikine VEGF ELISA.

Three independent runs for VEGF samples at $1.3 \mathrm{pM}$ and two independent runs for VEGF samples at $13 \mathrm{pM}$ were performed with the VEGF inhibitors. The mean percent analyte (VEGF) recovery $( \pm$ standard deviation 


\begin{tabular}{|c|c|c|c|c|}
\hline & Ligand & $\mathrm{k}_{\mathrm{a}} / 10^{5}\left(\mathrm{M}^{-1} \mathrm{~s}^{-1}\right)$ & $k_{d} / 10^{-5}\left(s^{-1}\right)$ & $K_{D}(p M)$ \\
\hline \multicolumn{5}{|l|}{ VEGF inhibitor [12] } \\
\hline Aflibercept & VEGF-A 165 & 410.0 & 2.01 & 0.49 \\
\hline Bevacizumab & VEGF-A 165 & 5.3 & 3.10 & 58 \\
\hline Ranibizumab & VEGF-A 165 & 1.6 & 0.73 & 46 \\
\hline \multicolumn{5}{|c|}{ R\&D systems quantikine VEGF capture mAb VEGF capture reagent [11] } \\
\hline R\&D-Clone 26503 & VEGF-A 165 & 270 & 3.81 & 1.41 \\
\hline
\end{tabular}

[SD]) for the experiments was calculated and plotted using a 4-Parameter Logistic nonlinear regression fit. The half-maximal inhibitory concentration $\left(\mathrm{IC}_{50}\right)$ for each VEGF inhibitor was also calculated (SoftMax Pro, Molecular Devices, LLC, CA, USA).

\section{Determination of VEGF:VEGF inhibitor complex detection in the quantikine VEGF ELISA}

The potential detection of VEGF bound to each of three VEGF inhibitors (aflibercept, ranibizumab and bevacizumab; VEGF:VEGF inhibitor complex) by the Quantikine VEGF ELISA was also examined. Samples containing $13 \mathrm{pM}(500 \mathrm{pg} / \mathrm{ml})$ of VEGF with increasing concentrations of each of the three VEGF inhibitors, ranging from 72.9 to $2.7 \mathrm{pM}$ in buffer, were incubated at room temperature for approximately 3 days. Samples were analyzed in the kit, however, at the detection step, the anti-VEGF polyclonal antibody from the kit was replaced with an anti-human $\operatorname{IgG~} \mathrm{Fc}_{\gamma}$ fragment-specific polyclonal antibody (for aflibercept and bevacizumab) or an anti-human $\left.\operatorname{IgG~} \mathrm{F}(\mathrm{ab})_{2}\right)_{2}$ fragment-specific polyclonal antibody (for ranibizumab).

Two independent runs for each VEGF inhibitor were performed. The mean response $( \pm S D)$ for the experiments was calculated.

\section{Results}

Binding affinity of the anti-VEGF 165 mAb

The $\mathrm{K}_{\mathrm{D}}(\mathrm{pM})$ of the putative Quantikine VEGF ELISA anti-VEGF 165 mAb for VEGF was determined to be $1.41 \mathrm{pM}$, which falls between the determined $\mathrm{K}_{\mathrm{D}}$ of aflibercept for VEGF $(0.49 \mathrm{pM})$ and that of ranibizumab (46 pM) and bevacizumab (58 pM; Table 1; the SPR sensorgrams are provided in Supplementary Figure 1). The binding affinity of this anti-VEGF $165 \mathrm{mAb}$ for VEGF is threefold weaker when compared with the affinity of aflibercept, but 33- to 40-fold stronger than the binding affinity of ranibizumab or bevacizumab, respectively. This large differential in VEGF-binding affinities between aflibercept and both ranibizumab and bevacizumab, relative to the binding affinity of the anti-VEGF $165 \mathrm{mAb}$ has the potential to disproportionately impact the detection of VEGF by the Quantikine VEGF ELISA in samples containing these inhibitors.

\section{VEGF inhibitor interference in the quantikine VEGF ELISA}

The impact of VEGF inhibitors on the detection of VEGF in the Quantikine VEGF ELISA was examined by preparing VEGF:VEGF inhibitor complexes at various molar ratios and assaying them using the Quantikine VEGF ELISA. Aflibercept, ranibizumab and bevacizumab all interfered with the measurement of VEGF concentrations in the Quantikine VEGF ELISA, as shown by the decrease in the recovery $(\% \pm S D)$ of VEGF $(1.3 \mathrm{pM})$ with increasing concentrations of each VEGF inhibitor (Figure 1). Aflibercept caused assay interference at much lower concentrations when compared with ranibizumab or bevacizumab. The $\mathrm{IC}_{50}$ values for the VEGF inhibitors (defined as the concentrations resulting in only 50\% VEGF recovery), at $1.3 \mathrm{pM} \mathrm{VEGF,} \mathrm{were} 0.45 \mathrm{pM}$ for aflibercept, compared with $24 \mathrm{pM}$ for ranibizumab and $138 \mathrm{pM}$ for bevacizumab. The observed degree of interference correlated with the relative binding affinities of the three VEGF inhibitors for VEGF.

At very low levels of all three VEGF inhibitors ( $\geq$ tenfold molar excess of VEGF), full recovery of VEGF was obtained using the Quantikine VEGF ELISA. Aflibercept began interfering with the Quantikine VEGF ELISA at VEGF concentrations that were in low molar excess (twofold molar excess of VEGF). Effectively, no VEGF was detected when aflibercept was in molar excess of VEGF. However, both ranibizumab and bevacizumab had to be present at much higher molar excess ( $\geq 62$-fold molar excess) to VEGF to cause a similar level of interference in 


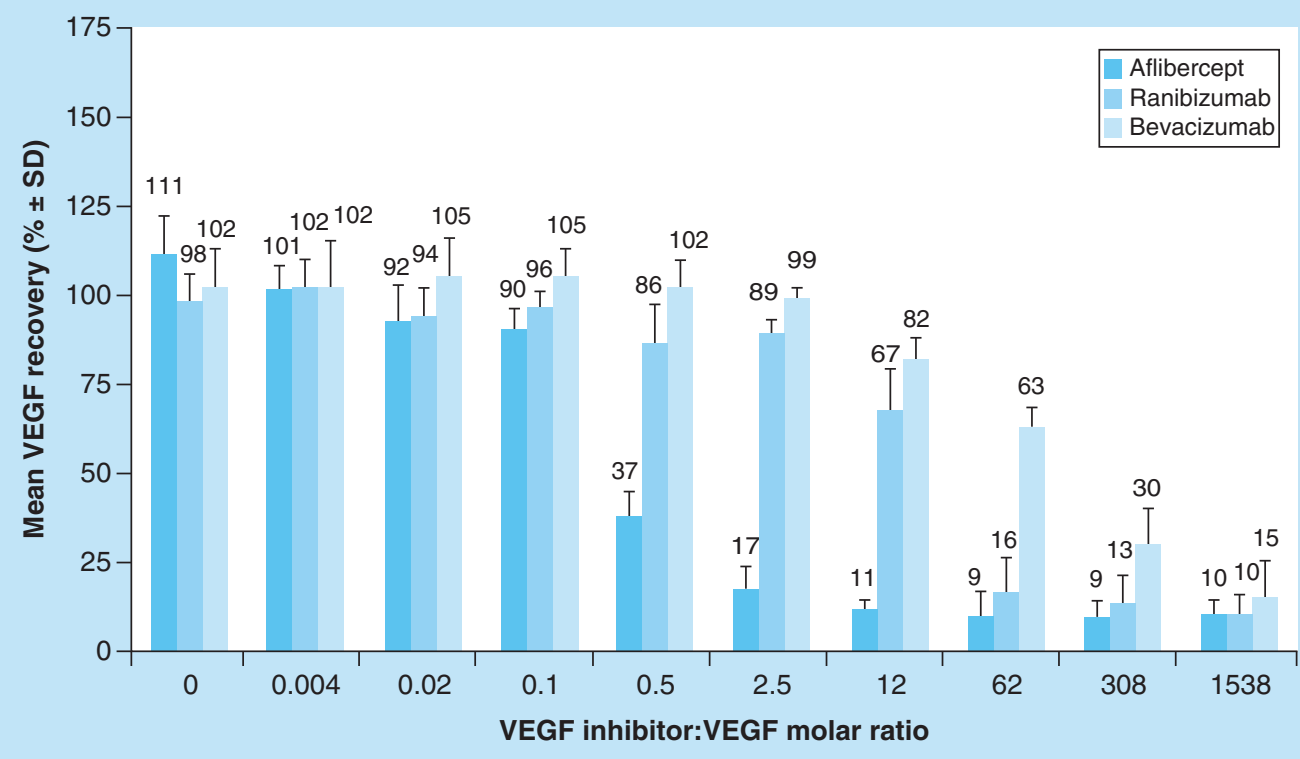

Figure 1. VEGF recovery from VEGF:VEGF inhibitor complexes following a 2-h plate incubation. Samples containing $1.3 \mathrm{pM}(50 \mathrm{pg} / \mathrm{ml})$ of VEGF with increasing concentrations of each inhibitor, ranging from 2000 to $0.005 \mathrm{pM}$, were prepared in buffer and incubated approximately 3 days before analysis using the Quantikine VEGF ELISA. Mean VEGF recovery $(\% \pm \mathrm{SD}$ ) was calculated from three independent runs. VEGF inhibitor molecular weights: aflibercept (100 kDa); ranibizumab (48 kDa); bevacizumab (149 kDa).

SD: Standard deviation.

the Quantikine VEGF ELISA. The level of interference of these VEGF inhibitors in the Quantikine VEGF ELISA appears to reflect their binding affinities $\left(\mathrm{K}_{\mathrm{D}}\right)$ for VEGF relative to the affinity of the capture antibody to VEGF.

VEGF recovery by the quantikine VEGF ELISA after extended sample incubation

The impact of incubation time on the results generated with the Quantikine VEGF ELISA in the presence of VEGF inhibitors was then examined by comparing results generated using the standard 2-h assay incubation to an overnight assay incubation. Aflibercept effectively competed with the Quantikine VEGF ELISA capture antibody, and extending the incubation time in the ELISA from $2 \mathrm{~h}$ (standard) to overnight had no significant impact on the recovery of VEGF in the presence of aflibercept (Figure 2). However, extending the incubation time significantly increased the recovery of VEGF in the presence of increasing concentrations of ranibizumab and allowed for full recovery of VEGF even in the presence of high molar excesses of bevacizumab. This suggests that not only does VEGF preferentially bind to the capture antibody rather than ranibizumab or bevacizumab but that, by allowing more time, even more of the VEGF will dissociate from these two VEGF inhibitors and bind to the ELISA capture antibody. A 2-h incubation time is insufficient for the ranibizumab and bevacizumab samples to reach equilibrium in this assay format. Interestingly, over-recovery of VEGF was consistently observed for both ranibizumab and bevacizumab complexes when the assay incubation times were extended. This was particularly pronounced in the ranibizumab experiments. This observed over-recovery in the presence of these two VEGF inhibitors is not understood but was reproducible, suggesting additional assay interference by both ranibizumab and bevacizumab, possibly due to detection of the VEGF:VEGF inhibitor complexes known to form with these two VEGF inhibitors.

\section{Detection of VEGF:VEGF inhibitor complexes by the quantikine VEGF ELISA}

A series of experiments were then conducted to determine whether the Quantikine VEGF ELISA detected VEGF complexed with the different VEGF inhibitors. Samples containing VEGF and the different inhibitors were prepared as previously described and added to the kit's plate. However, for experiments evaluating aflibercept or bevacizumab complexes, the kit's anti-VEGF detection antibody was replaced with a commercial anti-human IgG $\mathrm{Fc}_{\gamma}$ fragment-specific polyclonal antibody that would detect either aflibercept or bevacizumab in complex with VEGF. For experiments that evaluated ranibizumab complexes, the kit's anti-VEGF detection antibody was replaced 


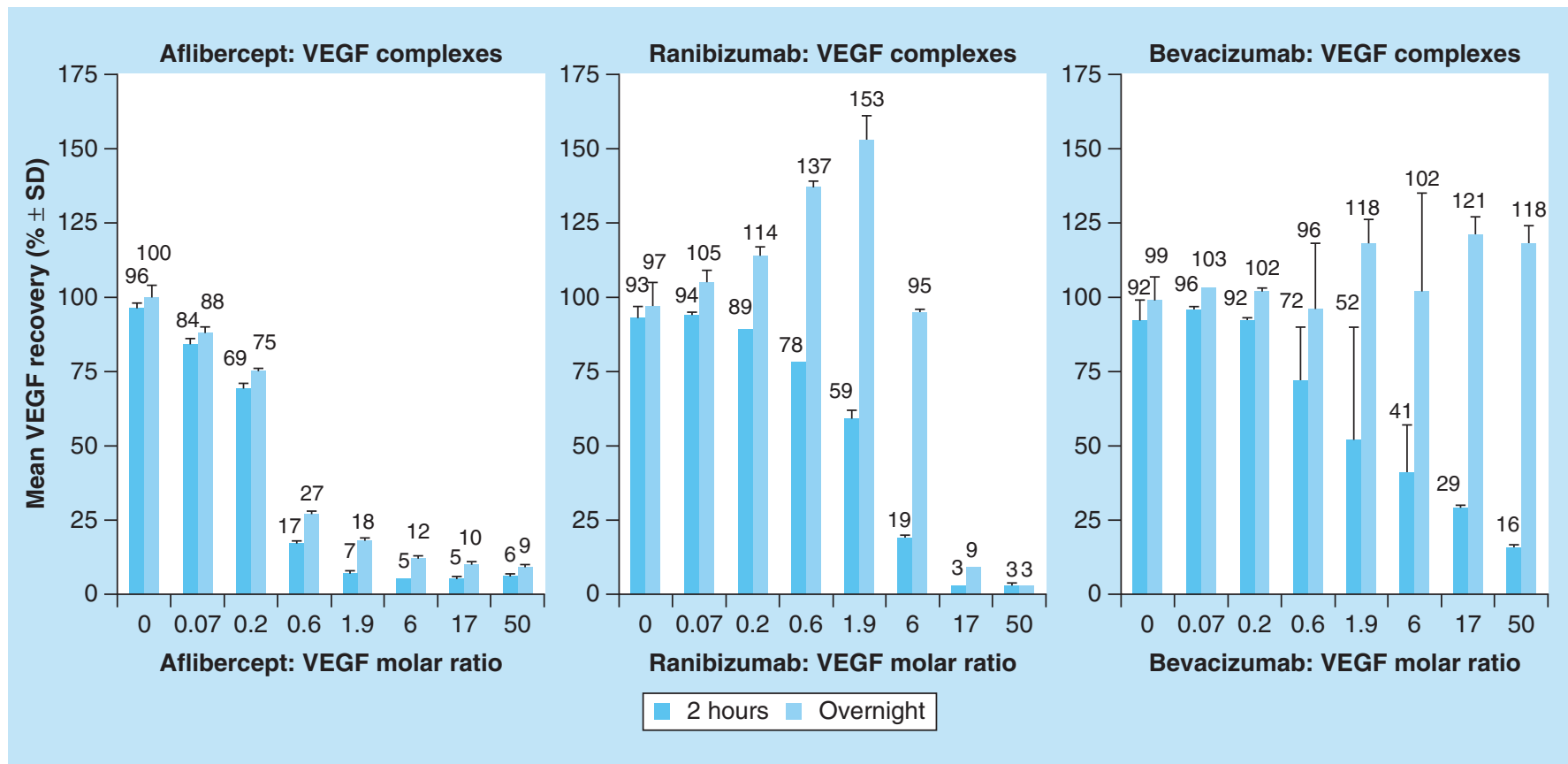

Figure 2. VEGF recovery from VEGF:VEGF inhibitor complexes following $\mathbf{2} \mathrm{h}$ or overnight plate incubation. Samples containing $13 \mathrm{pM}$ $(500 \mathrm{pg} / \mathrm{ml})$ of VEGF with increasing concentrations of each inhibitor, ranging from 656 to $0.9 \mathrm{pM}$, were prepared in buffer and incubated approximately 3 days before analysis using the Quantikine VEGF ELISA. Samples were analyzed following a 2-h sample incubation on the plate (normal kit conditions) or an extended overnight sample incubation ( $20 \mathrm{~h}$ ), at room temperature. Mean VEGF recovery (\% \pm SD) was calculated from two independent runs. VEGF inhibitor molecular weights: aflibercept (100 kDa); ranibizumab (48 kDa); bevacizumab (149 kDa). The range of inhibitor concentrations evaluated was 0 to 4000 pM (308 VEGF inhibitor:VEGF molar ratio). Results for inhibitor concentrations above the 50 molar ratio showed no significant change (results not shown).

SD: Standard deviation.

with a commercial anti-human $\operatorname{IgG} \mathrm{F}(\mathrm{ab})_{2}$ fragment-specific polyclonal antibody that would detect ranibizumab in complex with VEGF. In samples containing aflibercept:VEGF complexes, no VEGF:aflibercept complexes were detected in the assay (Figure 3). However, both ranibizumab and bevacizumab were detected in samples containing these VEGF inhibitors, indicating that VEGF:ranibizumab and VEGF:bevacizumab complexes were also being captured, rather than only free VEGF. The assay signal steadily increased as the relative molar excess of the VEGF inhibitor increased. The ranibizumab signal appeared to peak at around a twofold molar excess of ranibizumab over VEGF, but the bevacizumab signal continued to increase even at a sixfold molar excess of bevacizumab over VEGF (Figure 3). This increasing signal with bevacizumab in the assay may be due to the bivalent nature of both this drug and VEGF, which can lead to extended chains of bevacizumab and VEGF (i.e., paper dolls) [13].

\section{Discussion}

VEGF inhibitors, administered IVT, are used to treat multiple VEGF-related eye diseases. Systemic exposure to these VEGF inhibitors is minimal due to their route of administration and therefore is not expected to be clinically relevant. However, due to the relatively high affinities of each of the VEGF inhibitors for VEGF, it would be expected that any of these VEGF inhibitors in circulation would most likely be bound to VEGF. Several studies [14-17] reported that IVT administration of $2 \mathrm{mg}$ aflibercept decreased circulating free VEGF concentrations in plasma to a greater extent than IVT administration of $1.25 \mathrm{mg}$ bevacizumab or $0.5 \mathrm{mg}$ ranibizumab. These free VEGF concentrations were measured using a commercially available ELISA kit (Quantikine Human VEGF ELISA [R\&D Systems]), which has a lower limit of sensitivity of $9 \mathrm{pg} / \mathrm{ml}(0.23 \mathrm{pM})$ [11]. However, Takahashi et al. [18] reported that the Quantikine VEGF ELISA underestimated VEGF concentrations measured in a solution of known VEGF concentration $(1000 \mathrm{pg} / \mathrm{ml})$ in the presence of VEGF inhibitors, aflibercept, bevacizumab and ranibizumab, but not pegaptanib [18]. The mechanism or reason for this underestimation was not determined. A separate study [19], which used a different methodology to measure free VEGF (Bio-Plex Cytokine VEGF-A Luminex assay [Bio-Rad]), reported less impact on circulating VEGF after IVT administration of $2 \mathrm{mg}$ aflibercept, 


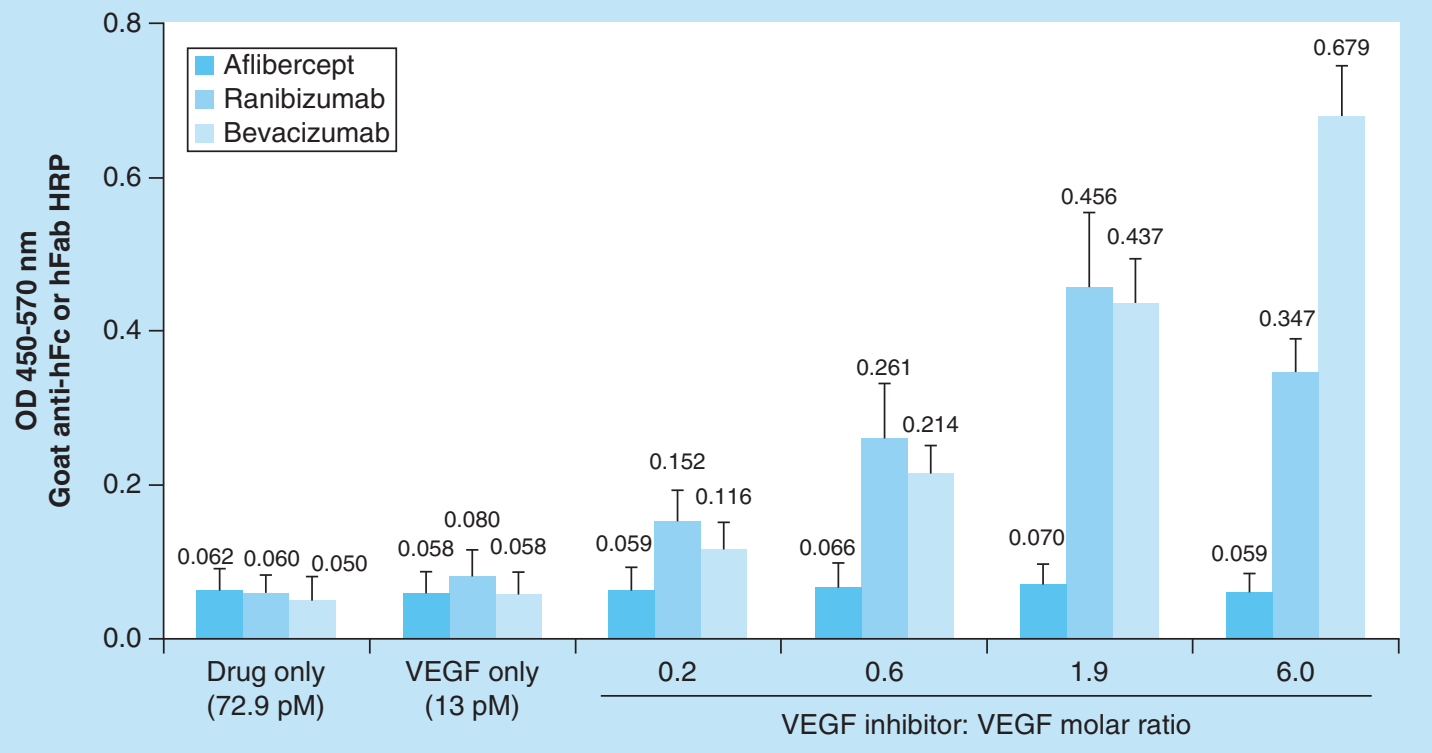

Figure 3. Detection of VEGF:VEGF inhibitor complexes in quantikine VEGF ELISA. Samples containing 13 pM $(500 \mathrm{pg} / \mathrm{ml})$ of VEGF with increasing concentrations of each inhibitor, ranging from 72.9 to $2.7 \mathrm{pM}$, were prepared and incubated at room temperature for approximately 3 days before analysis using the Quantikine VEGF ELISA. At the detection step, the anti-VEGF polyclonal antibody detection antibody in the kit was replaced with an anti-human IgG, Fc $\gamma$ fragment-specific, polyclonal antibody (for aflibercept and bevacizumab) or an antihuman IgG, F(ab') $)_{2}$ fragment-specific, polyclonal antibody (for ranibizumab). Mean OD $( \pm$ SD) was calculated from three independent runs. VEGF inhibitor molecular weights: aflibercept (100 kDa); ranibizumab (48 kDa); bevacizumab (149 kDa). OD: Optical density (450-570 nm); SD: Standard deviation.

demonstrating that different methods can lead to different results [18]. These two assay methodologies differed in assay type and format, with different working ranges for VEGF detection. However, both are subject to potential interference due to the presence of VEGF inhibitors in the sample, other format-related assay interferences, and the biochemical properties of the capture and/or detection reagents. To understand the disparity in these reported circulating free VEGF concentrations after IVT administration of aflibercept, an investigation was conducted to determine if VEGF inhibitors interfere in the Quantikine VEGF ELISA, impacting the ability of this assay to accurately measure free VEGF in the presence of these inhibitors.

The results of this investigation demonstrate that the Quantikine VEGF ELISA cannot accurately measure VEGF levels in plasma samples in the presence of VEGF inhibitors (Figure 1). The impact of these VEGF inhibitors on the detection of VEGF in the Quantikine VEGF ELISA was observed at two different VEGF concentrations: one approximating physiological levels of VEGF $(1.3 \mathrm{pM})$ and one tenfold higher $(13 \mathrm{pM})$. The results demonstrate that the level of assay interference is proportional to the binding affinities of each VEGF inhibitor for VEGF (Table 1). In the Quantikine VEGF ELISA, less VEGF is detected in the presence of aflibercept, which has the highest binding affinity for VEGF- $A_{165}\left(\mathrm{~K}_{\mathrm{D}}: 0.49 \mathrm{pM}\right)$, than is detected with a similar molar ratio of either ranibizumab $\left(\mathrm{K}_{\mathrm{D}}: 46 \mathrm{pM}\right)$ or bevacizumab $\left(\mathrm{K}_{\mathrm{D}}: 58 \mathrm{pM}\right.$; Figures $\left.2 \& 3\right)$. The relative differences in the binding affinities of the VEGF inhibitors and the putative Quantikine VEGF ELISA capture reagent, anti-VEGF $165 \mathrm{mAb}$, $\left(\mathrm{K}_{\mathrm{D}}: 1.41 \mathrm{pM}\right)$ are at least partially responsible for the differences in the detection of VEGF. Aflibercept has a higher binding affinity to VEGF than the capture reagent while both ranibizumab and bevacizumab have much weaker binding affinities for VEGF than the assay capture reagent. Thus, the Quantikine VEGF ELISA capture reagent can compete more efficiently for VEGF in the presence of these lower affinity VEGF inhibitors (Figure 4).

Experiments were also conducted to examine the effect of extended incubations on the Quantikine VEGF Assay. These extended incubation times had no effect on the detection of VEGF in the presence of aflibercept due to the higher binding affinity of aflibercept for VEGF relative to the affinity of the assay capture antibody for VEGF (Figure 2). However, the extended incubation times resulted in the detection of increased levels of VEGF even in the presence of excess molar concentrations of either ranibizumab or bevacizumab. This suggests that, over time, 
(A)

Assay in the absence of a VEGF inhibitor

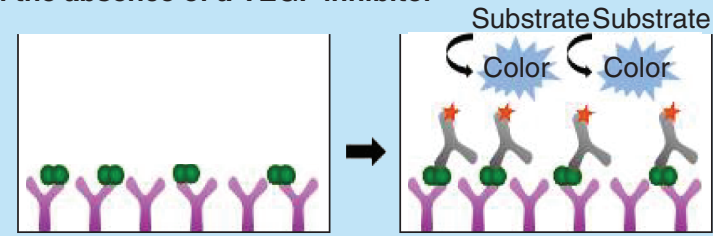

VEGF in the sample binds to the capture antibody and is detected.

(B) Assay in the presence of a VEGF inhibitor: before incubation, all VEGF is bound to the VEGF inhibitor

Aflibercept

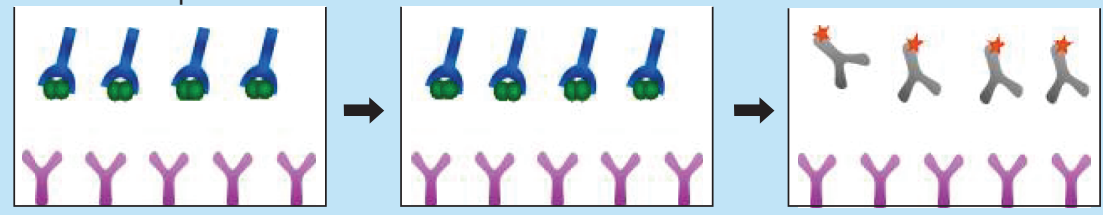

Due to high binding affinity, minimum dissociation from aflibercept occurs - no "free VEGF" is detected after incubation with detection antibody

Ranibizumab

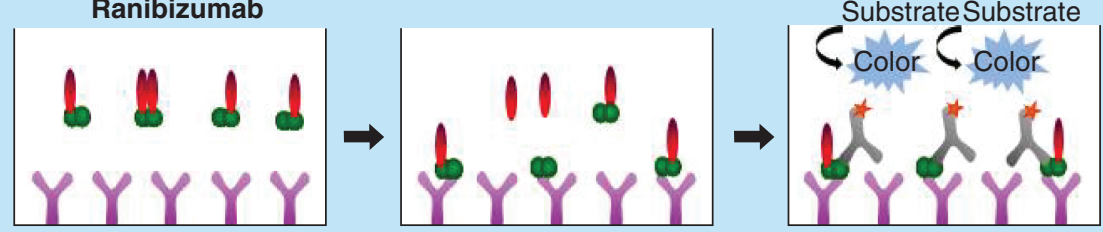

Due to the lower binding affinity, VEGF dissociates from ranibizumab, binding to the capture antibody and is detected as "free VEGF" after incubation with the detection antibody.

Ranibizumab:VEGF complexes are also captured and detected as "free VEGF" in the assay

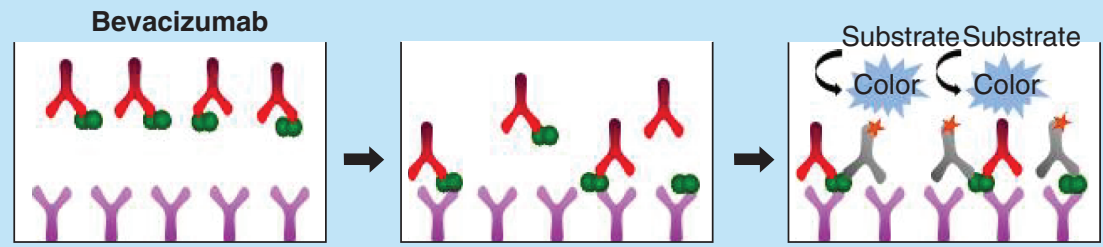

Due to the lower binding affinity, VEGF dissociates from bevacizumab, binding to the capture antibody and is detected as "free VEGF" after incubation with the detection antibody.

Bevacizumab:VEGF complexes are also captured and detected as "free VEGF" in the assay

Figure 4. Model of VEGF detection in sample containing either aflibercept, ranibizumab or bevacizumab using the quantikine VEGF ELISA.

due to the lower binding affinity of these VEGF inhibitors (compared with the assay capture antibody), VEGF is being released from VEGF:ranibizumab or VEGF:bevacizumab complexes and is being captured by the assay capture antibody. Interestingly, over-recovery of VEGF was observed under these extended incubation times for both ranibizumab and bevacizumab.

The over-recovery of VEGF in samples containing ranibizumab or bevacizumab, compared with samples containing aflibercept, raised the question of whether the Quantikine VEGF ELISA could also detect VEGF:VEGF inhibitor complexes, rather than just free VEGF. The potential detection of VEGF in complex with VEGF inhibitors would also impact the 'free' VEGF concentrations being reported using the Quantikine VEGF Assay.

Experiments were then conducted to determine whether VEGF:VEGF inhibitor complexes for each of the three VEGF inhibitors were being captured in the Quantikine VEGF ELISA. The results demonstrated that for both ranibizumab and bevacizumab, VEGF:VEGF inhibitor complexes are being captured by the Quantikine capture antibody and the VEGF in these complexes is being detected by the assay (Figure 3). This is possible because VEGF 
is a homodimer, with two binding sites for both ranibizumab and bevacizumab. Each of these VEGF inhibitors (an anti-VEGF antibody and an Fab antibody fragment) can only bind to one site on one of the VEGF subunits at a time, leaving the other VEGF subunit free to either bind to the Quantikine capture antibody or to another ranibizumab or bevacizumab molecule. Thus, for ranibizumab and bevacizumab, both free VEGF and VEGF complexed to these inhibitors are captured and detected by the Quantikine VEGF assay (Figure 4). Aflibercept, on the other hand, is a fusion protein and contains multiple VEGF receptor domains, which bind simultaneously to both VEGF subunits. Thus, VEGF bound to aflibercept cannot bind to the Quantikine capture antibody and the complex is not detected by the Quantikine VEGF assay.

The pattern of the VEGF:VEGF inhibitor complex binding observed for both ranibizumab and bevacizumab in the Quantikine VEGF ELISA is consistent with the formation of large multimeric complexes between bevacizumab and VEGF, as described by MacDonald et al. [13]. In contrast, aflibercept forms homogenous 1:1 complexes with VEGF that are not detected by the Quantikine VEGF ELISA. The assay signal generated by ranibizumab complexes increases with increasing concentrations of ranibizumab up to an approximately twofold molar excess and then decreases, while the assay signal generated by the bevacizumab complexes continues to increase with increasing concentrations of bevacizumab (Figure 2). These observed results are attributed to the formation of large multimeric complexes with VEGF due to how these molecules bind to VEGF. Because ranibizumab is a Fab fragment, it can only bind one of the VEGF subunits to its VEGF binding site, forming a 1:1 complex with VEGF at low molar ratios of ranibizumab. As the concentration of ranibizumab increases, the concentration of 1:1 VEGF:ranibizumab complexes in the sample increases. However, these complexes can still be captured in the Quantikine ELISA due to the dimeric nature of VEGF (since ranibizumab is still only bound to one of the subunits, leaving the other subunit available for binding to the kit capture antibody; Figure 3). However, as the concentrations of ranibizumab increase to greater than equimolar, 1:2 VEGF:ranibizumab complexes can form. With ranibizumab bound to both subunits, these 1:2 VEGF:ranibizumab complexes cannot bind to the Quantikine capture antibody and the level of these captured complexes detected in the assay decreases. Bevacizumab, on the other hand, is an intact mAb, capable of binding two VEGF molecules at the same time. At low molar ratios of bevacizumab, 1:1 and some 2:1 complexes of VEGF:bevacizumab are formed, with bevacizumab binding one or two distinct VEGF molecules. At higher molar ratios of bevacizumab, higher order VEGF:bevacizumab complexes (paper dolls) can form. These higher order structures can potentially bind to the Quantikine capture antibody, resulting in ever increasing concentrations of this complex being captured.

\section{Conclusion}

The results presented here indicate that the differences between VEGF concentrations observed using the Quantikine VEGF ELISA after administration of aflibercept, ranibizumab or bevacizumab mostly reflect the differences in their binding affinities for VEGF relative to the affinity of the Quantikine VEGF ELISA capture reagent, as well as the mechanism of how these VEGF inhibitor molecules actually bind to VEGF. All three VEGF inhibitors, when administered IVT, are present at very low levels in circulation and bind to circulating VEGF to form complexes. The use of the R\&D Systems Quantikine VEGF ELISA to measure 'free' VEGF in circulation, in the presence of VEGF inhibitors, is problematic, since, as demonstrated, the presence of VEGF inhibitors directly interferes with the capture and detection of VEGF by the assay. The level of this interference is dependent on the relative binding affinities of each VEGF inhibitor. Therefore, comparisons between levels of 'free' VEGF in the presence of these different inhibitors cannot be made since variable but artifactual assay results would be obtained. This is especially evident for ranibizumab and bevacizumab (weaker VEGF binders than the ELISA kit's capture antibody). Over time, bound VEGF is released from VEGF complexes with ranibizumab and bevacizumab and detected in the kit as 'free' VEGF, even in the presence of excess inhibitor. This overestimation of 'free' VEGF is not seen with aflibercept, which binds to VEGF with a higher binding affinity than the ELISA kit's capture antibody.

Furthermore, VEGF:VEGF inhibitor complexes formed with both ranibizumab and bevacizumab are also detected by the Quantikine VEGF ELISA, amplifying the overestimation of the levels of 'free' VEGF with these two inhibitors and further distorting the results of the analysis. This does not occur with aflibercept. Thus, the reported decreases in circulating VEGF concentration following IVT administration of aflibercept [14-17], when compared with ranibizumab and bevacizumab, as determined by the Quantikine VEGF ELISA assay, are unreliable. These results are an artifact due to the higher binding affinity of aflibercept for VEGF relative to these other VEGF inhibitors, the unique mechanism of how aflibercept binds to VEGF and the ability of the kit to actually detect VEGF:ranibizumab and VEGF:bevacizumab complexes as well as free VEGF. 


\section{Future perspective}

It is anticipated that these findings will discourage use of the R\&D Quantikine VEGF ELISA kit to measure free VEGF in the presence of VEGF inhibitors and may lead to the development of improved and more sensitive assays, which are not prone to VEGF inhibitor interference and are capable of accurately quantitating free VEGF in biological samples in the presence of VEGF inhibitors.

\section{Summary points}

\section{Background}

- To examine if Quantikine human VEGF isoform $165\left(\right.$ VEGF $\left._{165}\right)$ ELISA kit (R\&D Systems) accurately quantitates free VEGF $_{165}$ in samples containing VEGF inhibitors aflibercept, ranibizumab and bevacizumab.

- To understand the mechanism of interference by VEGF inhibitors in the measurement of VEGF 165 using the Quantikine VEGF ELISA. This understanding is necessary for the correct interpretation of results from previous publications [4,14-17], which have reported VEGF levels measured in clinical samples containing VEGF inhibitors using the Quantikine VEGF ELISA.

- Understanding that the challenges presented by VEGF inhibitors in the measurement of VEGF 165 is relevant for the conduct of future research with samples that contain VEGF inhibitors.

\section{Methods}

- Binding affinity of the anti-VEGF 165 mAb (MAB293; R\&D Systems), the putative capture antibody for the Quantikine VEGF ELISA, for VEGF was determined by surface plasmon resonance.

- VEGF inhibitor interference with VEGF quantification was evaluated by incubating VEGF with increasing concentrations of each inhibitor.

- VEGF recovery using the kit was determined after the standard $2 \mathrm{~h}$ or overnight incubation.

- Potential detection of VEGF:VEGF inhibitor complexes by the kit was evaluated by replacing the kit's polyclonal anti-VEGF antibody with antibodies specific to human IgG.

Results \& discussion

- Binding affinity of the anti-VEGF 165 , the putative capture antibody for the Quantikine VEGF ELISA, was 1.41 pM, threefold weaker than aflibercept, but 33- and 40-fold stronger than ranibizumab or bevacizumab, respectively.

- Experiments confirmed that due to this relative binding disparity, aflibercept interferes with the detection of VEGF in the Quantikine VEGF ELISA to a greater extent than either ranibizumab or bevacizumab.

- Extending the incubation time from $2 \mathrm{~h}$ to overnight did not affect VEGF recovery with aflibercept but substantially increased it with ranibizumab and bevacizumab, indicating VEGF dissociation and subsequent binding by the kit's capture antibody.

- Using antibodies recognizing VEGF inhibitors at the detection step showed the kit also detected VEGF:ranibizumab and VEGF:bevacizumab complexes but not VEGF:aflibercept complexes.

\section{Conclusion}

- Quantikine VEGF ELISA kit (R\&D Systems) cannot accurately quantitate plasma VEGF levels in the presence of these VEGF inhibitors as they interfere differentially with the capture and detection of free VEGF.

\section{Acknowledgements}

The authors thank Susan Wilt for her outstanding efforts in preparing the manuscript and figures. The authors also thank our colleagues at Regeneron Pharmaceuticals, Inc. and Bayer HealthCare for their critical review of this manuscript and their helpful comments and suggestions.

Financial \& competing interests disclosure

All the authors are employees of Regeneron Pharmaceuticals, Inc., and report stock ownership for Regeneron Pharmaceuticals, Inc. This study was funded by Regeneron Pharmaceuticals, Inc., NY, USA. The authors have no other relevant affiliations or financial involvement with any organization or entity with a financial interest in or financial conflict with the subject matter or materials discussed in the manuscript apart from those disclosed.

No writing assistance was utilized in the production of this manuscript.

Open access

This work is licensed under the Attribution-NonCommercial-NoDerivatives 4.0 Unported License. To view a copy of this license, visit http://creativecommons.org/licenses/by-nc-nd/4.0/ 


\section{References}

Papers of special note have been highlighted as: $\bullet \bullet$ of considerable interest

1. Kaiser PK, Kodjikian L, Korobelnik JF et al. A systemic pharmacokinetic/pharmacodynamic analysis of intravitreal aflibercept injection in patients with retinal diseases. BMJ Open Ophthalmol.(2018) (In Press).

2. Crawford Y, Ferrara N. VEGF inhibition: insights from preclinical and clinical studies. Cell Tissue Res. 335(1), 261-269 (2009).

3. Bressler SB. Introduction: understanding the role of angiogenesis and antiangiogenic agents in age-related macular degeneration. Ophthalmology 116(10 Suppl.), S1-S7 (2009).

4. Aiello LP, Avery RL, Arrigg PG et al. Vascular endothelial growth factor in ocular fluid of patients with diabetic retinopathy and other retinal disorders. N. Engl. J. Med. 331(22), 1480-1487 (1994).

5. Eylea [package insert]. Tarrytown, NY: Regeneron Pharmaceuticals, Inc. (2014).

6. Lucentis [package insert]. South San Francisco, CA: Genentech, Inc. (2013).

7. Avastin [package insert]. South San Francisco, CA: Genentech, Inc. (2015).

8. Economides AN, Carpenter LR, Rudge JR et al. Cytokine traps: multi-component, high-affinity blockers of cytokine action. Nat. Med. 9, 47-52 (2003)

-• Describes the creation of high-affinity cytokine traps, including aflibercept (VEGF-Trap), and their function as high affinity blockers of cytokine function.

9. Rudge JS, Holash J, Hylton D et al. VEGF Trap complex formation measures production rates of VEGF, providing a biomarker for predicting efficacious angiogenic blockade. Proc. Natl Acad. Sci. USA 104(47), 18363-18370 (2007).

10. George ML, Eccles SA, Tutton MG, Abulafi AM, Swift RI. Correlation of plasma and serum vascular endothelial growth factor levels with platelet count in colorectal cancer: clinical evidence of platelet scavenging? Clin. Cancer Res. 6(8), 3147-3152 (2000).

11. Quantikine VEGF ELISA [package insert] Minneapolis, MN: R\&D Systems, Inc. (2016).

12. Papadopoulos N, Martin J, Ruan Q et al. Binding and neutralization of vascular endothelial growth factor (VEGF) and related ligands by VEGF Trap, ranibizumab and bevacizumab. Angiogenesis 15(2), 171-185 (2012).

-. Presents the characterization of the binding affinities for the VEGF inhibitors aflibercept, bevacizumab and ranibizumab. The online version contains supplemental information that provides the technical details for determination of the $K_{D}$ values.

13. MacDonald DA, Martin J, Muthusamy KK et al. Aflibercept exhibits VEGF binding stoichiometry distinct from bevacizumab and does not support formation of immune-like complexes. Angiogenesis 19, 389-406 (2016).

-. Describes the VEGF binding stoichiometry exhibited by aflibercept is distinct from that observed with bevacizumab, whose binding stoichiometry supports formation of extended chain complexes.

14. Zehetner C, Kralinger MT, Modi YS et al. Systemic levels of vascular endothelial growth factor before and after intravitreal injection of aflibercept or ranibizumab in patients with age-related macular degeneration: a randomised, prospective trial. Acta Ophthalmol. 93(2), e154-e159 (2015).

-• Presents free VEGF levels in the presence of VEGF inhibitors using the Quantikine human VEGF-A ELISA kit (R\&D Systems).

15. Avery RL, Castellarin AA, Steinle NC et al. Systemic pharmacokinetics following intravitreal injections of ranibizumab, bevacizumab or aflibercept in patients with neovascular AMD. Br. J. Ophthalmol. 98(12), 1636-1641 (2014).

-. Presents free VEGF levels in the presence of VEGF inhibitors using the Quantikine human VEGF-A ELISA kit (R\&D Systems).

16. Yoshida I, Shiba T, Taniguchi $\mathrm{H}$ et al. Evaluation of plasma vascular endothelial growth factor levels after intravitreal injection of ranibizumab and aflibercept for exudative age-related macular degeneration. Graefes Arch. Clin. Exp. Ophthalmol. 252(9), 1483-1489 (2014).

-. Presents free VEGF levels in the presence of VEGF inhibitors using the Quantikine human VEGF-A ELISA kit (R\&D Systems).

17. Wang X, Sawada T, Sawada O, Saishin Y, Liu P, Ohji M. Serum and plasma vascular endothelial growth factor concentrations before and after intravitreal injection of aflibercept or ranibizumab for age-related macular degeneration. Am. J. Ophthalmol. 158(4), 738-744, e731 (2014).

-• Presents free VEGF levels in the presence of VEGF inhibitors using the Quantikine human VEGF-A ELISA kit (R\&D Systems).

18. Takahashi H, Nomura Y, Nishida J, Fujino Y, Yanagi Y, Kawashima H. Vascular endothelial growth factor (VEGF) concentration is underestimated by enzyme-linked immunosorbent assay in the presence of anti-VEGF drugs. Invest. Ophthalmol. Vis. Sci. 57, 462-466 (2016).

-• Demonstrated that VEGF levels in the presence of VEGF inhibitors aflibercept, bevacizumab and ranibizumab are underestimated by the Quantikine human VEGF-A ELISA kit (R\&D Systems).

19. Roald AB, Aass HC, Moe MC. Recovery of plasma vascular endothelial growth factor concentrations during aflibercept loading phase and after the transition to bimonthly treatment for neovascular age-related macular degeneration. Br. J. Ophthalmol. 99(12), 1610-1613 (2015).

-• Presents free VEGF levels in the presence of VEGF inhibitors using a Luminex method, which generates different results from the R\&D kit. 
